\title{
Psicología y salud en México: algunas reflexiones basadas en el sentido común y la experiencia*
}

\section{Psychology and Health In Mexico: Some Reflections Based on Common Sense and the Experience}

\author{
Julio Alfonso Piña López ** \\ Universidad de Sonora, México \\ Claudia Sánchez-Bravo \\ Instituto Nacional de \\ Perinatología, México \\ Ismael García-Cedillo \\ Universidad Autónoma de San \\ Luis Potosí, México \\ José Luis Ybarra Sagarduy \\ Universidad Autónoma de \\ Tamaulipas, México \\ Cirilo H. García Cadena \\ Universidad Autónoma de \\ Nuevo León, México \\ Recibido: 4 de marzo de 2013 \\ Revisado: 13 de mayo de 2013 \\ Aceptado: 31 de junio de 2013
}

\section{Resumen}

A mediados de la pasada década se publicó un trabajo en el que se analizaba por qué, a pesar del desarrollo experimentado por la psicología y salud en México, en ese entonces se hacían evidentes dos problemas: la falta de reconocimiento profesional de la psicología y los psicólogos en los marcos normativos sobre la materia, por un lado, así como las dificultades que enfrentaban para su integración en los equipos interdisciplinarios de salud, por el otro. Casi una década después nos encontramos que en general la situación no ha cambiado de manera sustancial, lo que a final de cuentas se traduce en un ejercicio profesional que dista mucho de estar impactando positivamente al diseño, instrumentación y evaluación de programas de investigación e intervención en el seno mismo de las instituciones de salud. Con base en el sentido común y la experiencia de los autores, en este trabajo se discute sobre tres asuntos que consideramos pudieran ayudar a revertir dicha situación, en beneficio de la psicología y los psicólogos que se desempeñan en el ámbito de la salud.

Palabras clave: psicología y salud, interdisciplina, investigación, intervención, enfermedades crónicas.

\footnotetext{
** Correspondencia: Julio Alfonso Piña, Programa de Salud Institucional, Universidad de Sonora, México. Guillermo Prieto No. 18. Colonia Constitución. Hermosillo, Sonora, C.P. 83150 (México). Correo electrónico: jp131260@yahoo.com.mx.
} 


\section{Abstract}

In the middle of the last decade was published a work in which analyzed why, despite the development experienced by the psychology and health in Mexico, then became evident two problems: the lack of professional recognition of psychology and psychologists in the normative frameworks on the subject, on the one hand, as well as the difficulties faced by these for their integration into the interdisciplinary teams of health, on the other. Almost a decade later we find that overall the situation has not changed substantially, which ultimately translates into a professional practice that is far be positively impacting the design, implementation and evaluation of research and intervention programs in the context of health institutions. Based on common sense and the experience of the authors, in this work is discussed on three issues that we believe could help to reverse this situation, for the benefit of psychology and psychologists working in the health field.

Keywords: health psychology, interdisciplinary, research, intervention, chronic diseases.

\section{Introducción}

En la presentación de una sección especial publicada en la revista Health Psychology titulada “¿Qué nos traerá el futuro?”, Keefe y Blumenthal (2004) concluyeron: a. la psicología y salud se había desarrollado rápidamente en los últimos 25 años y se le podría concebir como un campo profesional establecido; b. los psicólogos de la salud compartían una frontera de trabajo común con otros investigadores y clínicos, por lo que se encontraban bien posicionados para realizar importantes contribuciones en el futuro, y c. no obstante lo anterior, se requería mejorar la formación y el entrenamiento de los psicólogos de la salud.

Aun cuando en general coincidimos con los autores, al menos en países como el nuestro se impone hoy en día una necesaria y más amplia discusión sobre el contenido de cada punto; considérese para tal fin lo siguiente:

1. En México, infortunadamente la psicología y los psicólogos carecemos de representación formal en los marcos normativos sobre la materia, sobre todo si se tiene en cuenta que lo relacionado con la prevención, curación y rehabilitación de las enfermedades se concibe como competencia exclusiva de los profesionales de la medicina, según lo consignado en los artículos 32 y 33 de la Ley General de Salud (Piña, 2004); dicha situación, también infortunadamente, no había cambiado entrada la presente década (Piña, 2011).

2. Con todo y que frecuentemente se apela al discurso y al quehacer interdisciplinario cuando se conduce una investigación o un programa de intervención, es importante hacer notar que los esfuerzos en esa dirección se practican en buena medida desde los institutos nacionales de salud; destacan, entre otros, los de cancerología (Olvera-Manzanilla, Ruiz-González, Platas de la Mora, Ochoa-Carrillo y Alvarado-Aguilar, 2011), pediatría (Garduño, Ham, Méndez, Rodríguez, Díaz y Reyes, 2009; Garduño y Méndez, 2010) y perinatología (Meza-Rodríguez, Mancilla-Ramírez, Sánchez, Figuero-Damián, Contreras-Maldonado y Carreño-Meléndez, 2011).

3. Los programas de formación y entrenamiento en psicología y salud, en ambos niveles, licenciatura y posgrado, salvo sus muy honrosas excepciones se distinguen por su incongruencia e incoherencia en los dominios teórico-metodológico y en el de la aplicabilidad del conocimiento psicológico; es decir, entre lo que se "enseña" con lo que se presume se "entrena" (Ribes, 2011; Sánchez-Sosa, 2008). De ahí que sean programas que no proveen los recursos competenciales - saber qué y saber cómo-que faciliten la incorporación de los futuros psicólogos a los equipos interdisciplinarios de salud. 


\section{El estatuto de la psicología y salud en México como campo de actuación profesional}

Si se tiene en cuenta que la psicología y salud en México empezó a adquirir forma y algunos vestigios de organización a partir de la década de los ochenta del pasado siglo, hoy día cabría esperar que fuese ya un campo de actuación profesional plenamente establecido; la realidad, dicho sea de paso, es que estamos lejos de haber alcanzado esta condición. En efecto, dando por descontado el acercamiento que, por ejemplo, ha tenido el programa doctoral de la Facultad de Psicología de la Universidad Nacional Autónoma de México con las instituciones de salud por medio de sus programas de residencia, o bien la existencia de otros tantos orientados a la investigación-intervención que se han venido creando en los diferentes institutos nacionales de salud entre quienes se autonombran psicólogos de la salud, no existe ni acuerdo ni consenso sobre diferentes asuntos que, a nuestro modo de ver, resultan de capital importancia. Concretamente tres, que nos proponemos revisar por separado y sobre los cuales, en la parte final de este trabajo, ofrecemos una visión de conjunto.

\section{¿Qué es psicología de la salud y cómo promover su consolidación profesional en el entramado social y en las instituciones del ramo?}

Con frecuencia, los psicólogos de la salud en México suelen retomar algunas definiciones sobre el campo de actuación que provienen de distintas fuentes institucionales o de autores en particular -i.e., American Psychological Association, Friedman y DiMatteo, Matarazzo, Stone, Taylor, etcétera-, a las que hacen adecuaciones ad hoc. Se concluye asegurando, por ejemplo, que el psicólogo de la salud es un profesional capacitado para realizar actividades que se enmarcan en los rubros de evaluación, diagnóstico, prevención, explicación, tratamiento/modificación de problemas físicos/mentales o de cualquier otra conduc- ta que sea relevante para la salud y la enfermedad (véanse Cantú y López, 2009; Oblitas, 2008; Ortiz, 1996).

Por principio de cuentas, habría que aclarar que los psicólogos de la salud no diagnostican -algo que por simple lógica sí hacen los médicos-, ni mucho menos explican. En efecto, la de diagnóstico constituye una categoría que se inscribe dentro de la lógica del modelo médico y su correspondiente apoyo en criterios morfológicos, con base en los cuales presumiblemente se "describe" o se "da cuenta" de la "sintomatología" que manifiesta una persona en la forma de "angustia", "ansiedad”, “estrés”, “dolor”, “depresión”, etc., que, por supuesto, no son entidades equiparables a un tumor maligno, un infarto del miocardio, la diabetes o la infección por el VIH. Pero tampoco explican porque ésta no constituye una competencia profesional, de las que sí se podrían incluir evaluar, planear, prevenir, investigar e intervenir (Reidl, 2008; Ribes, 2005, 2006; Sánchez-Sosa, 2009).

\section{¿Qué es, entonces, psicología y salud?}

Para responder a esta pregunta es preciso, por una cuestión elemental y de sentido común, responder a la pregunta de qué es psicología. Ésta, sin apellidos o extensiones -i.e., clínica, de la salud, educativa, ambiental, comunitaria, del trabajo, etc.-, es simple y sencillamente una disciplina en proceso de consolidación como empresa científica. Luego entonces, algo que por principio de cuentas debería quedar claro para el colectivo de psicólogos es que primero está la psicología y luego todas las psicologías con sus apellidos o extensiones, las habidas o por haber.

Para emplear una metáfora, diríamos que la psicología es la "madre" de todas esas extensiones profesionales, por lo que no se puede concebir ni definir la psicología y salud justificando su existencia a partir de otras psicologías, que se distinguen justo por su naturaleza "aplicada"; así, Stone (1988), en Estados Unidos, la concibe como una de las subdivisiones de la psicología clínica; Martín (2003), en Cuba, como derivación de la psicología clínica, la médica, la comunitaria y 
hasta de la medicina conductual; finalmente, Álvarez, Moral de la Rubia y Martínez (2009) y Oblitas (2008), en México, dependiendo de la clínica, la comunitaria y la social, principalmente.

El punto sobre el que ponemos énfasis es que en la medida en que se tenga claridad sobre qué es, y por tanto, cómo definir la psicología, es en la misma medida en que posteriormente se tendrá claridad para hacer lo propio respecto de la psicología y salud. Si aquí entendemos por psicología una disciplina de conocimiento que tiene que ver con cómo, por qué y en qué circunstancias las personas se comportan como lo hacen, para responder a estas u otras preguntas lo que se requiere es una teoría psicológica general o de procesos (Ribes); citaremos al autor en extenso:

El conocimiento científico [...] se construye en la forma de enunciados causales -o mejor dicho explicativos- a partir de la formulación de enunciados fácticos sobre ocurrencias. El proceso teórico consiste en cómo inferir de situaciones de hecho particulares a otras situaciones de hecho particulares, cómo explicar situaciones de hecho particulares refiriéndolas a otras situaciones de hecho, y cómo producir o prevenir estados de cosas que garanticen dichas situaciones de hecho. (2009, p. 6)

Tal y como se desprende de esta cita, hay que entender que el conocimiento científico obtenido en y mediante la práctica de la investigación no se traslada de manera directa del laboratorio a los escenarios institucionales o naturales donde cobra sentido su posterior aplicación; antes se requiere un proceso especial de traducción y que se disponga de las categorías conceptuales pertinentes para predecir, con un grado razonable de certeza, cuán probable es que una persona se comportará de tal o cual manera en determinada circunstancia, según lo haya hecho en el pasado en circunstancias funcionalmente similares (Ibáñez, 2007; Piña, Fierros, García e Ybarra, 2011).

Predecir, en el sentido de identificar las condiciones en las que eventualmente algo sucederá o acontecerá (Real Academia Española, 1985), es resultado de una diversidad de actividades que se enmarcan en un plano distinto, aunque complementario del científico: el profesional. Luego entonces, toda vez que el conocimiento científico no se puede aplicar de manera directa, invariablemente requiere su traducción en la forma de conocimiento tecnológico o aplicado, que es relevante y adquiere significación en el segundo de los planos: el profesional. Retomando a Ribes sobre esto último apunta:

El conocimiento aplicado, entendido como conocimiento tecnológico o conocimiento práctico, se construye y ejercita de manera distinta al conocimiento científico. El conocimiento aplicado no sólo se fundamenta parcialmente en la experiencia directa con los objetos y acontecimientos particulares, sino que se dirige a esos mismos objetos y acontecimientos, ya sea en la forma de predicciones sobre su comportamiento, o bien en la forma de operaciones de manipulación e intervención directa o indirecta. $(2009$, p. 8)

Y justo a partir de lo antes expuesto es donde está el nodo de la discusión. La psicología como disciplina de conocimiento se funda en la premisa de que las personas nos comportamos de determinada manera y en determinadas circunstancias, para cuyo entendimiento se precisa de una teoría general de procesos, que permitiría describir y explicar. Por consiguiente, nótese que se ponen en cursivas los fines que persigue cualquier teoría psicológica general de procesos, por lo que, por razones obvias, no podríamos esperar que en la psicología y salud se explique algo, en el mismo sentido en el que se explica en aquélla.

De ahí que esta diferencia aparentemente banal entre los fines que persiguen una teoría psicológica general de procesos sea fundamental para entender por qué en el contexto particular de la relación psicología y salud es fundamental: a. contar con modelos teóricos debidamente articulados en lo conceptual; b. que sean susceptibles de ser probados y validados en los distintos escenarios sociales, y c. que provean los elementos mínimos para generar conocimiento tecnológico o aplicado para ser utilizado en aquellos escenarios en los que se requiere trabajar con la conducta de las personas, ya sea promoviendo las que se vinculan 
con el cuidado y mantenimiento de la salud, bien modificando las de riesgo por las de prevención, o bien facilitando las que son pertinentes para el ajuste a la enfermedad y los tratamientos, fundamentalmente.

Los modelos teóricos, en virtud de que no surgen de la nada, no pueden concebirse como invenciones discursivas al margen de: a. una definición de lo psicológico; b. considerar qué categorías de análisis son pertinentes; c. cuáles son los conceptos en los que se apoyarán, y d. cómo se relacionarán con los fenómenos de la salud y la enfermedad (Piña, Ybarra, Alcalá y Samaniego, 2010). Como bien apunta Ibáñez (2007), haciendo alusión a la teoría de la conducta de Ribes y López (1985) en tanto que sistema comprensivo o ciencia básica: aun cuando ésta posee algunos elementos para abordar los eventos psicológicos, sus operaciones e interrelaciones, carece a la vez de otros tantos para aproximarse a la especificidad de los fenómenos sociales de interés, que tradicionalmente se les concibe como problemas sociales -i.e., salud-. Esos elementos no son otros que lo que aquél da en llamar como categorías "puente" o "interfase", que median el contacto del investigador con el lenguaje teórico del sistema comprensivo.

Dichas categorías, cuyo ejemplo conspicuo se tiene con la de personalidad, vendrían a imprimirle un sentido de consistencia al discurso teóricoconceptual en relación con los fenómenos de la salud y la enfermedad. Sirven como "puente" o "interfase", en tanto que, como ya se comentó, las categorías de y para el análisis de lo psicológico en el ámbito básico no se pueden trasladar arbitrariamente al ámbito aplicado, porque ambas comprenden compromisos distintos y operan, por simple lógica, en ámbitos también distintos.

\section{El problema con lo expuesto a lo largo de este apartado es que en México se ha venido operando sobre la base de dos ideas equívocas}

1. Al margen de qué se entiende por psicología como disciplina de conocimiento, una vez que se retoman algunas definiciones sobre psicolo- gía de la salud o se hacen adecuaciones ex profeso a estas, es común que los autores terminen concibiendo la psicología como una disciplina de conocimiento que es distinta y distante de la psicología como profesión, aunque paradójicamente se asegure que la psicología de la salud: a. concentra las aportaciones científicas y tecnológicas generadas desde diferentes campos de la psicología (Ortiz, 1996), o b. que constituye un enfoque novedoso dentro de la psicología contemporánea, en el cual se tiene perfectamente identificado cómo operan los principales factores biopsicosociales sobre la salud y la enfermedad (Oblitas, 2004). Que, ciertamente, ambas psicologías sean distintas no conlleva que sean distantes, puesto que por una lógica elemental la segunda no puede existir al margen de o con independencia de la primera.

2. Luego entonces, si la psicología y salud deviene, se nutre $y$, por consiguiente, su existencia tiene sentido, metafóricamente hablando, como "hija" de la psicología en tanto que disciplina de conocimiento, aquélla tiene que definirse y ubicarse en el marco de los subsistemas aplicados a los que alude Ibáñez (2007). Su compromiso es el de proveer a los profesionales de la psicología los recursos competenciales - de nuevo, saber qué y saber cómo- que les permitan incidir eficientemente sobre los problemas de salud.

Finalmente, ¿qué debemos entender entonces por psicología y salud? De acuerdo con un planteamiento que se hizo desde mediados de la pasada década, como un:

Campo de actuación profesional en que los psicólogos poseen tanto los conocimientos -saber acerca de las cosas- como las habilidades y destrezas - saber hacer las cosas de manera eficiente- indispensables para cumplir con las funciones de investigación, prevención y rehabilitación, fundamentalmente, con especial énfasis en la investigación de qué y cómo las variables psicológicas facilitan o dificultan la práctica de las conductas instrumentales de riesgo o prevención, con el objeto de prevenir una enfermedad y promover la salud. (Piña y Rivera, 2006, p. 673) 
Ciertamente, aun cuando en su momento los autores pusieron especial énfasis en la prevención de las enfermedades y la promoción de la salud, de ello no se colige que las actividades vinculadas con la rehabilitación, por ejemplo, pasen a un segundo plano. No, partiendo del entendido de que en México, y en virtud de la conocida como transición epidemiológica que se originó desde hace casi tres décadas, entre las principales causas de mortalidad general se encuentran las enfermedades del corazón y la diabetes, que se distinguen de otras tantas en la medida en que la dimensión psicológica - ergo, la conducta- ha demostrado desempeñar un papel clave previo a su acaecimiento y su eventual rehabilitación (Reynoso, Álvarez, Tron, de la Torre y Seligson, 2002; Ybarra y Samaniego, 2010).

\section{¿Es el de psicología y salud un campo de actuación por definición interdisciplinario?}

Si la psicología en el ámbito básico estudia la conducta de las personas en interacción con objetos, eventos y otras personas en los medios físico-químico, ecológico y social, en el de la aplicabilidad del conocimiento psicológico lo que interesa es predecir en qué circunstancias es probable que aquellas practiquen determinadas conductas que se presume guardan relación -como en el caso que nos ocupa- con el cuidado, mantenimiento, pérdida y eventual recuperación de la salud. Para este último propósito es necesario que se disponga de las categorías tipo puente o interfase, que permitan vincular ambos niveles, así como lo psicológico, con los fenómenos de la salud y la enfermedad.

Empero, como habrá de notarse, ambas actividades se sitúan en el contexto de la psicología como disciplina y profesión, esto es, en los niveles "básico" y “aplicado", respectivamente. No nos dicen nada acerca de si, en el segundo de los niveles, y hablando en concreto de la psicología y salud, ésta comporta un campo de actuación por definición interdisciplinario que se integra con otros en un ámbito específico de problemas sociales, ergo, el de salud.
Retomando los planteamientos de Ribes (2009), puesto que las profesiones - psicología y salud, psicología clínica, educativa, ambiental, del trabajo o de las organizaciones, etc.- no constituyen disciplinas formales, desde el punto de vista del conocimiento son interdisciplinas. En este ámbito se encuentran, por ejemplo, la medicina, la enfermería, el trabajo social y, claro está, la psicología y salud; todos son campos de actuación profesionales que se integran y sintetizan en un ámbito específico de problemas sociales.

Ciertamente, como sostiene Ribes (2011), las diferentes interdisciplinas carecen de teoría, al menos en los términos en que se le entiende en el contexto de la psicología como disciplina o en proceso de consolidación como empresa científica: como un cuerpo articulado de categorías y conceptos dirigidos a identificar o reconocer las formas genéricas de interacción entre un organismo y el ambiente, que operan en distintos ámbitos de complejidad (Ribes y López, 1985). Si, como se comentó en el apartado previo, el conocimiento generado en el ámbito básico no se traslada ni aplica directamente en el ámbito aplicado, entonces ¿qué conocimiento se aplica y, sobre todo, cómo se aplica?

Se aplica el conocimiento científico una vez que se "traduce" en la forma de conocimiento tecnológico o práctico, para hacerlo extensivo a los escenarios institucionales -hospitales, clínicas, etcétera- o naturales -comunidades, hogar- en los que justo cobra relevancia. Para ello se requiere, siguiendo esta lógica, contar con modelos teóricos que permitan "bajar la cortina" y relacionar las categorías generales con su potencial aplicabilidad, adaptándolas a personas concretas, sobre problemas concretos y en circunstancias también concretas. Por consiguiente, es requisito indispensable contar con un marco teórico que haga posible traducir el conocimiento científico por definición, analítico- en conocimiento tecnológico - por definición, sintético y sintetizador(Ribes y López, 1985). Por tanto, el conocimiento psicológico es aplicable, siempre y cuando se haga en relación con la persona comportándose en lo individual y en circunstancias específicas: en aquellos ámbitos en los que la dimensión psicológica es clave. 
Siguiendo este precepto, el de psicología y salud, que se inscribe en el ámbito de aplicabilidad potencial del conocimiento psicológico, es decir, como profesión, constituye por tanto una interdisciplina que, si bien comparte con otras tantas un mismo encargo social -el de la salud-, se distingue de todas ellas porque aporta un conjunto de categorías y conceptos para el estudio de la dimensión psicológica que es pertinente a la salud y la enfermedad, así como un conjunto de procedimientos y técnicas para promover la salud, prevenir el acaecimiento de una enfermedad o procurar su rehabilitación. Por tanto, el de psicología y salud se sitúa en el mismo plano que otras profesiones, cuyo común denominador es el ámbito de la salud (figura 1).

No obstante, y como acertadamente apunta Ribes (2005), el meollo del asunto estriba en entender que a la par del conocimiento que se aplica está el cómo se aplica. Ello puede ocurrir de dos maneras: apoyándose en los profesionales de alguna interdisciplina -i.e., médicos, enfermeras, trabajadoras sociales, promotores de salud-o en los propios usuarios de los servicios -i.e., líderes comunitarios, grupos de apoyo, pacientes y sus familiares-. Aquí, es condición necesaria y suficiente integrar la psicología y salud en la agenda de la salud pública (Arrivillaga, 2009; Londoño, Valencia y Vinaccia, 2006), lo que en principio presupone que el rol del psicólogo incluye: a. la de ser un experto que transmite o transfiere el conocimiento a otros profesionales, ya sea que ello ocurra a lo largo de su proceso de formación o en su interacción cotidiana en el seno mismo de las instituciones del sector, entiéndase hospitales, clínicas y centros de salud, principalmente, y b. a los propios usuarios de los servicios.

Así mismo, comprende la de ser un experto en el diseño y evaluación de los mejores procedimientos para el cambio conductual, que comprende identificar y evaluar los problemas, plantear las soluciones pertinentes y evaluar posteriormente su idoneidad, efectividad y eficacia (Michie y Abraham, 2004; Ribes, 2009); en síntesis, evaluar el impacto de los programas (Scopeta, 2006).

Una propuesta en esa dirección se ha hecho recientemente (Piña, 2012) para el cabal cumplimiento de esos roles en el contexto de los centros ambulatorios para la prevención y atención a VIH/ sida e ITS en México, definiendo, a partir de la que se denominó una ruta crítica, los tres momentos clave que se encuentran implicados una vez que una persona es diagnosticada como seropositiva al VIH, pasando por la evaluación inicial de si se requiere un plan de intervención, para culminar con su eventual diseño, instrumentación y evaluación de seguimiento. En estos tres momentos es fundamental que los diferentes profesionales de salud sepan qué hacer, cómo y por qué hacer las cosas (esto es, competencialmente), considerando a la par una diversidad de funciones que les son propias: diagnosticar, informar, instruir, entrenar, regular disposiciones, etc.

Tal y como se consigna en el siguiente apartado, este doble rol entraña por supuesto diferentes retos, que se relacionan tanto con el perfil profe-

Figura 1. Relaciones entre diferentes interdisciplinas en el ámbito de la salud

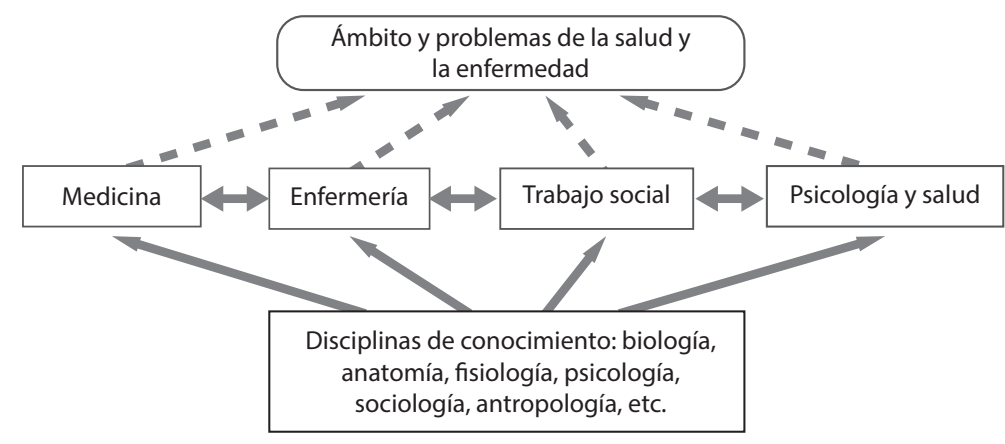


sional del psicólogo que se desempeñará profesionalmente en el ámbito de la salud y, en especial, con las competencias que son necesarias para los propósitos de incidir eficientemente sobre las enfermedades crónicas epidemiológica y socialmente relevantes.

\section{¿Qué se requiere para formar psicólogos competentes para incidir eficientemente sobre las enfermedades crónicas más apremiantes?}

Formar psicólogos competentes no es una tarea fácil. En países como el nuestro, en el que se asegura existen alrededor de 400 programas de licenciatura tanto en universidades públicas como en privadas, es posible que hayan egresado más de 100 mil psicólogos (Ribes, 2011). Como bien señala el autor, la cuestión - de forma y fondo- radica en saber si toda esa amplia gama de programas se distinguen por: a. su identidad disciplinaria y b. contar con el personal calificado en investigación básica y aplicada, a la vez que debidamente entrenado en la aplicabilidad del conocimiento psicológico en los distintos ámbitos: salud, educación, ambiente, convivencia social, etc.

No está por demás mencionar que, en relación con la identidad disciplinaria, esta cobra importancia y sentido si se le inscribe dentro de una orientación y una lógica curricular. Prosiguiendo con Ribes, la filosofía curricular que se ha adoptado en la inmensa mayoría de universidades en México ha sido la del eclecticismo, que se caracteriza por: a. confundir diferentes posiciones como si fueran complementarias; b. sustentarse en el supuesto de que la información transmitida del docente al alumno es equiparable al conocimiento que se ha acumulado, y c. confundir los campos de conocimiento con los campos de aplicación.

Siendo así, el docente -quien presumiblemente conoce- "enseña" sobre las múltiples escuelas o corrientes existentes en psicología, las distintas teorías y sus contenidos, etc., apoyándose en una práctica eminentemente verbalista en y con la cual "transmite" al alumno aquello que conoce. Éste, por extensión, se presume que "aprende" sobre todo ello, sin que necesariamente entre en contacto con lo que se le se debería enseñar en situaciones observacionales o experimentales (Irigoyen, Jiménez y Acuña, 2007). Así mismo, al confundirse los campos de conocimiento -las teorías que tratan con la fenomenología de lo psicológico- con propuestas orientadas a la aplicación del conocimiento psicológico en el ámbito de la salud, se asume equívocamente que las teorías se "aplican" directamente sobre los problemas propios de dicho ámbito.

Luego entonces, la fragrante ausencia en la identidad disciplinaria, tarde o temprano se ve acompañada de la ausencia manifiesta de un perfil profesional perfectamente definido, que sea claro tanto en sus propósitos como en sus objetivos. De ahí que, como refiere Ribes (op. cit., p. 89): De hecho, el perfil profesional es siempre el perfil específico de una identidad disciplinaria. No es lógico suponer que identidades disciplinarias distintas puedan converger en un mismo criterio social de inserción y el perfil profesional correspondiente.

Por tan elementales razones, en las actuales circunstancias y, salvo sus muy honrosas excepciones, los programas de formación de los psicólogos en México podría decirse que mantienen esa correspondencia lógica entre la identidad disciplinaria y el perfil profesional de egreso, cuyos ejemplos conspicuos se tienen con el programa de licenciatura en Psicología de la Universidad de Sonora y los de posgrado que se ofertan en el Centro de Estudios e Investigaciones en Comportamiento de la Universidad de Guadalajara, en las facultades de Psicología de la Universidad Nacional Autónoma de México, Autónoma de Nuevo León y Autónoma de San Luis Potosí, así como en la Unidad de Trabajo Social y Ciencias para el Desarrollo Humano de la Universidad Autónoma de Tamaulipas.

Habida cuenta de lo antes dicho, ¿cómo trascender el actual estado de cosas, de cara a garantizar una formación disciplinaria sólida, que se vea aparejada de un perfil profesional que también garantice el entrenamiento de las competencias que son pertinentes al ámbito y los problemas de 
la salud y la enfermedad? Una primera respuesta conlleva evitar a toda costa el eclecticismo en la psicología, del que podríamos decir, metafóricamente hablando, que es el "padre" de todas las inconsistencias discursivas en torno a la fenomenología de lo psicológico. Por consiguiente, se requiere una psicología robusta en lo teóricoconceptual, que la haga corresponder con los métodos para el estudio de lo psicológico - procedimientos y preparaciones experimentales, así como con el diseño de sistemas observacionales y de registro- y generadora de un cuerpo tecnológico - procedimientos y técnicas- que nos permita cumplir con las tareas de evaluación e intervención en los escenarios naturales e institucionales.

Debería quedar claro al lector, siguiendo con Ribes (2011), que si se carece de un cuerpo científico básico de conocimientos, no se puede generar un cuerpo de conocimientos potencialmente aplicable a los problemas de interés, caso de la salud. Adicionalmente, es requisito fundamental tener claro qué se espera del psicólogo en el contexto de las propias instituciones de salud, pues resulta ilógico e incomprensible definir un perfil profesional si se desconoce cuáles son las necesidades más apremiantes de las instituciones del sector -llámense institutos nacionales de salud, hospitales de zona, hospitales generales, clínicas, centros ambulatorios o centros de salud-y las de las comunidades, que ubicaríamos para fines descriptivos como escenarios institucionales y naturales, respectivamente (figura 2).

Figura 2. Componentes básicos por considerar en el trabajo del psicólogo en el ámbito de la salud

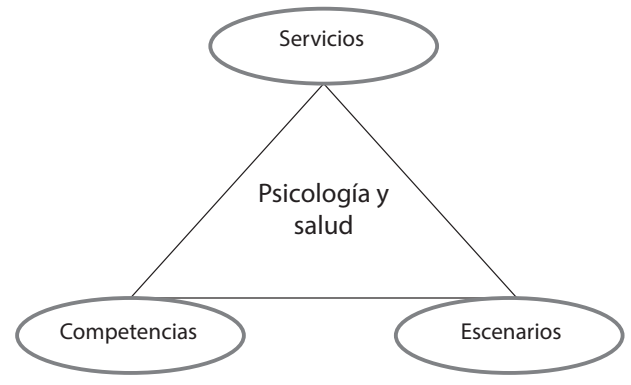

Si pensáramos, por ejemplo, en la necesidad de formar y entrenar a los psicólogos en competencias que incluyen la evaluación, planeación, prevención, investigación e intervención -con fines de rehabilitación o para los cuidados paliativos-, está perfectamente demostrado que algunas de ellas pueden ser particularmente pertinentes en algunas de esas instituciones y otras en las comunidades. En las primeras cabría la posibilidad de que la evaluación, investigación e intervención adquieran primacía (Linden, Moseley y Erskine, 2005), mientras que en las segundas lo serían las de evaluación, planeación y prevención, con un enfoque propio de lo que se conoce como atención primaria a la salud (Durán, Hernández y Becerra, 1995).

Todas ellas, no obstante, precisan de conocimientos y habilidades específicas (tabla 1). Destacan por su importancia conocimientos que tienen que ver con psicología, la definición de lo psicológico como objeto de estudio, la delimitación de la dimensión psicológica que es pertinente a la salud y la enfermedad, los modelos teóricos y sus características conceptuales, los conceptos de salud y enfermedad, las políticas públicas en materia de salud y su relación con la psicología y lo psicológico, los métodos - procedimientos, sistemas de observación y registro- por utilizar, etc. (García, 2009; García y Piña, 2012; Sánchez-Sosa, 1998).

Las habilidades comprenderían, entre otras cosas, la evaluación de necesidades individuales, familiares y/o comunitarias como requisito previo para el diseño, instrumentación y evaluación de programas preventivos, de rehabilitación o de cuidados paliativos; el diseño y puesta en marcha de programas de investigación; el diseño y puesta en marcha de programas de intervención en las comunidades, para interactuar efectiva y eficientemente con otros profesionales de la salud (Sánchez-Sosa, 2008, 2009).

Finalmente, sobre esto último es importante llamar la atención respecto a un problema referido años atrás por Reidl (2008), quien señala que solo pueden entrenar las competencias profesionales en el ámbito de la salud quienes han demostrado pericia y competencia. Ya que las competencias 
Tabla 1.

Conocimientos y habilidades/destrezas en psicología y salud

\begin{tabular}{|c|c|c|}
\hline Conocimientos & Habilidades/destrezas & Logros \\
\hline $\begin{array}{l}\text { 1. Qué es psicología y cuál es el } \\
\text { objeto de estudio. } \\
\text { 2. Qué teorías psicológicas } \\
\text { existen y cuáles son sus } \\
\text { fundamentos epistémicos y } \\
\text { ontológicos. } \\
\text { 3. Qué categorías y conceptos } \\
\text { son pertinentes en relación } \\
\text { con la fenomenología de lo } \\
\text { psicológico. } \\
\text { 4. En qué consiste la dimensión } \\
\text { psicológica en la salud y la } \\
\text { enfermedad. } \\
\text { 5. Qué modelos teóricos existen } \\
\text { y cuáles son sus características } \\
\text { distintivas en lo conceptual. } \\
\text { 6. De qué métodos } \\
\text { (procedimientos, } \\
\text { preparaciones experimentales, } \\
\text { sistemas de observación y } \\
\text { registro) se dispone para la } \\
\text { evaluación, investigación e } \\
\text { intervención. } \\
\text { 7. Cómo relacionar los modelos } \\
\text { teóricos, sus categorías y } \\
\text { conceptos con los métodos de } \\
\text { que se dispone. } \\
\text { 8. Cómo se elaboran, qué } \\
\text { contienen y cómo se evalúan } \\
\text { las políticas públicas en } \\
\text { materia de salud. }\end{array}$ & $\begin{array}{l}\text { 1. Diseñar estrategias prototipo } \\
\text { para la evaluación y } \\
\text { planeación en los escenarios } \\
\text { institucionales y naturales. } \\
\text { 2. Administrar proyectos. } \\
\text { 3. Diseñar, instrumentar y evaluar } \\
\text { la efectividad y eficiencia de } \\
\text { programas de prevención e } \\
\text { intervención. } \\
\text { 4. Diseñar, instrumentar y evaluar } \\
\text { la pertinencia y viabilidad de } \\
\text { programas de investigación. } \\
\text { 5. Interactuar eficientemente con } \\
\text { otros profesionales de la salud. } \\
\text { 6. Interactuar eficientemente } \\
\text { con líderes e integrantes de } \\
\text { comunidades. }\end{array}$ & $\begin{array}{l}\text { 1. Aplicación del conocimiento } \\
\text { psicológico en relación con la } \\
\text { salud y la enfermedad: para } \\
\text { prevenir el acaecimiento de } \\
\text { una enfermedad, promover } \\
\text { la salud y participar en } \\
\text { la rehabilitación de una } \\
\text { enfermedad o en los cuidados } \\
\text { paliativos. } \\
\text { 2. Generación de nuevo } \\
\text { conocimiento psicológico } \\
\text { en relación con la salud y la } \\
\text { enfermedad. } \\
\text { 3. Desarrollo de procedimientos, } \\
\text { sistemas de observación y } \\
\text { registros. } \\
\text { 4. Desarrollo y validación de } \\
\text { paquetes tecnológicos para el } \\
\text { cambio conductual. }\end{array}$ \\
\hline
\end{tabular}

se entrenan en la práctica, se requiere que en los programas de formación y entrenamiento de los psicólogos de la salud el personal responsable esté debidamente calificado para fungir como "modeladores" y "supervisores" de lo que los estudiantes harán una vez que se inserten en los escenarios institucionales o naturales. Ello presupone, antes que otra cosa, que ese personal se sujete a evaluaciones periódicas que garanticen su certificación y el ejercicio profesional con oportunidad, calidad y sentido ético.

De ahí que en nuestro país sea hoy día indispensable hacer un alto en el camino, reflexionar críticamente en torno de si los programas académicos en psicología están cumpliendo con dicho cometido $\mathrm{y}$, sobre todo, si quienes se autonombran psi- cólogos de la salud son competentes o no para los propósitos de entrenar, modelar y supervisar in situ a los futuros psicólogos de la salud.

\section{Algunas reflexiones y apuntes finales}

Una reflexión sobre el futuro de la psicología de la salud y de su abordaje en instituciones de salud no psiquiátricas (campo rico por desarrollar por el psicólogo de la salud) en México nos lleva a proponer un par de puntos que consideramos fundamentales y que pueden ayudar a consolidar su quehacer cotidiano. En primer lugar, es necesario que los psicólogos dispongan de los recursos competenciales relacionados con la psicología como disciplina de conocimiento y como profesión; hay que conocer 
sobre qué es psicología, qué es psicología y salud, pero, sobre todo, tener claridad en torno a cómo opera la dimensión psicológica que es pertinente en la salud y la enfermedad.

En segundo lugar, y en el supuesto de que los psicólogos dispongan de esos recursos competenciales, no hay que pasar por alto que, en el caso de las personas que acuden a las instituciones para la atención de una enfermedad -i.e., cáncer, infarto del miocardio, diabetes, insuficiencia renal, infección por el VIH-, una pregunta elemental que habría que responder sería la siguiente: ¿cómo desarrollar propuestas de trabajo que permitan consolidar la presencia y la participación decisiva del psicólogo en los equipos interdisciplinarios de salud dentro de las instituciones?

Nótese que ponemos especial énfasis en el quehacer profesional de los psicólogos en las instituciones, porque es justo en éstas en las que por razones históricas la incorporación de aquéllos ha sido restringida, lo cual se da más bien de manera gradual y con marcadas limitaciones para cumplir con funciones que trasciendan las tradicionales, por ejemplo, la de evaluación. En efecto, ya que las enfermedades crónicas más apremiantes en el país demandan la puesta en marcha de programas interdisciplinarios, el equipo de psicólogos en las instituciones debe ofrecer una intervención psicológica integral de alta calidad - para prevenir el acaecimiento de las diferentes enfermedades crónicas, pero, sobre todo, para los fines que llevan a su rehabilitación-, que se distinga por ser el producto de líneas claras sobre cuándo, cómo y por qué intervenir, así como propuestas de investigación que les permitan retroalimentar y mejorar esos programas de intervención (figura 3).

Son propuestas que deben contribuir a incrementar y/o mejorar los conocimientos respecto a la propia enfermedad y el manejo de los pacientes para hacerle frente. Así como el médico no puede basarse únicamente en su juicio clínico, el psicólogo debe basar sus propuestas de intervención en los hallazgos obtenidos en la práctica de investigación, cuyo objetivo es dar cuenta de los fenómenos psicológicos y la forma como operan en el curso clínico de cualquier enfermedad cró-
Figura 3. Modelo de un programa integral en instituciones de salud no psiquiátricas

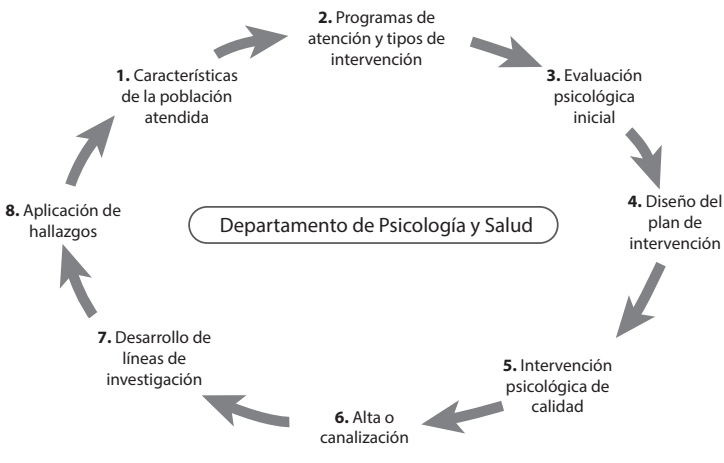

nica, entendiendo: ¿quién va a intervenir? ¿Con qué objetivo? ¿Cuáles son las necesidades de las personas que han recibido el diagnóstico de una enfermedad crónica? ¿Qué aspectos de lo propiamente psicológico se deben considerar en las investigaciones? Y, finalmente, ¿cómo traducir los hallazgos en propuestas de intervención?

Son interrogantes necesarios que requieren respuestas puntuales, que confiamos sentarán las bases para la creación y consolidación de Departamentos de Psicología y Salud -independientes de los de Psiquiatría y que, por tanto, se conformen con base en sus propios criterios profesionales- dentro de las instituciones del ramo, que se distingan por la claridad de sus objetivos, la definición puntual de las funciones que son pertinentes y el auspicio de líneas de trabajo que articulen la investigación con la intervención.

Finalmente, disponer de las competencias teóricas y metodológicas en psicología, por un lado, y en psicología y salud, por el otro, aderezadas ambas con una dosis apropiada de sentido común y con la experiencia que se va generando en el campo de actuación, bien podría ayudar a revertir el actual estado de cosas, a sentar las bases para auspiciar y consolidar un proyecto profesional genuino e independiente de las profesiones biomédicas y sociales, pero que a la vez garantice su incorporación al conjunto de profesiones que dan vida y sentido al quehacer interdisciplinario. 


\section{Referencias}

Álvarez, J., Moral de la Rubia, J., \& Martínez, J. (2009). Prólogo. La psicología en la promoción de la salud. Retos del rol de las psicólogas y psicólogos de la salud. En J. Álvarez, J. Moral de la Rubia y J. Martínez (Comps.), Psicología e investigación en enfermedades degenerativas crónicas (pp. 7-12). Monterrey, México: Universidad Autónoma de Nuevo León.

Arrivillaga, M. (2009). Psicología y salud pública: tensiones, encuentros y desafíos. Universitas Psychologica, 8, 137-148.

Cantú, R., \& López, G. (2009). Psicología de la salud: antecedentes, aplicaciones y propuestas. Monterrey, México: Universidad Autónoma de Nuevo León.

Durán, L. I., Hernández, M., \& Becerra, J. (1995). La formación del psicólogo y su papel en la atención primaria a la salud. Salud Pública de México, 37, 462-471.

García, C. H. (2009). Cómo investigar en psicología. México: Trillas.

García, C. H., \& Piña, J. A. (2012). Políticas públicas en materia de salud en México, con especial énfasis en el periodo 1994-2000. Salud y Sociedad: Investigaciones en Psicología Social y de la Salud, 3, 194-209.

Garduño, A., Ham, O., Méndez, J., Rodríguez, J., Díaz, E., \& Reyes, C. (2009). Experiencias en cuidados paliativos en el Instituto Nacional de Pediatría. Revista Mexicana de Pediatría, 76, 75-80.

Garduño, A., \& Méndez, J. (Eds.) (2010). Guía práctica de cuidados paliativos en pediatría. Atención integral en la fase terminal. México: Instituto Nacional de Pediatría/Corinter.

Ibáñez, C. (2007). Problemas de aplicación social del conocimiento disciplinario de la psicología interconductual. Acta Comportamentalia, 15, 81-92.
Irigoyen, J. J., Jiménez, M. Y., \& Acuña, K. F (2007). Aproximación a la pedagogía de la ciencia. En J.J. Irigoyen, M.Y. Jiménez y K.F. Acuña (Eds.), Enseñanza, aprendizaje y evaluación (pp. 13-44). Hermosillo, México: Universidad de Sonora/Consejo Nacional de Ciencia y Tecnología.

Keefe, F. J., \& Blumenthal, J. A. (2004). Health psychology: What will be the future bring? Health Psychology, 23, 156-157.

Linden, W., Moseley, J., \& Erskine, W. (2005). Psychology as a health-care profession: Implications for training. Canadian Psychology, 46, 179-188.

Londoño, C., Valencia, S. C., \& Vinaccia, S. (2006). El papel del psicólogo en la salud pública. Psicología y Salud, 16, 199-205.

Martín, L. (2003). Aplicaciones de la psicología en el proceso salud-enfermedad. Revista Cubana de Salud Pública, 29, 275-281.

Meza-Rodríguez, M. P., Mancilla-Ramírez, J., Sánchez-Bravo, C., Figuero-Damián, R., Contreras-Maldonado, L. E., \& Carreño-Meléndez, J. (2011). Respuesta psicológica en la infección por virus de inmunodeficiencia humana. Perinatología y Reproducción Humana, 25, 236240.

Michie, S., \& Abraham, C. (2004). Interventions to change health behaviours: Evidence-based or evidence-inspired? Psychology and Health, 19, 29-49.

Oblitas, L. A. (2004). Prefacio. En L.A. Oblitas (Coord.), Psicología de la salud y calidad de vida (p. XVII). México: Thomson.

Oblitas, L. A. (2008). El estado del arte de la psicología de la salud. Revista de Psicología, XXXVI, 220-254.

Olvera-Manzanilla, E., Ruiz-González, D. S., Platas-de la Mora, A., Ochoa-Carrillo, F. J., \& Alvarado-Aguilar, S. (2011). Aspectos neuropsicológicos de los pacientes con tumores 
cerebrales. Gaceta Médica de Oncología, 10, 143-149.

Ortiz, G. R. (1996). Psicología y medicina: viejos problemas, nuevas perspectivas. En G. R. Ortiz (Comp.), Psicología y salud. La experiencia mexicana (pp. 17-39). Xalapa, México: Universidad Veracruzana.

Piña, J. A. (2004). La psicología y los psicólogos de la salud en México: algunas realidades perturbadoras. International Journal of Clinical and Health Psychology, 4, 191-205.

Piña, J. A. (2010). El rol del psicólogo en el ámbito de la salud: de las funciones a las competencias profesionales. Enseñanza e Investigación en Psicología, 15, 233-255.

Piña, J. A. (2011). Psicología y salud: obstáculos y posibilidades para su desarrollo en el siglo XXI. Bogotá: PSICOM Editores.

Piña, J. A. (2012). Adhesión en personas con VIH: una visión crítica desde la psicología. Madrid: Editorial Académica Española.

Piña, J. A., Fierros, L. E., García, C. H., \& Ybarra, J. L. (2011). Psicología y salud (II): tendiendo puentes entre la psicología básica y la aplicada: el rol del fenómeno de personalidad. Pensamiento Psicológico, 9, 203-212.

Piña, J. A., \& Rivera, B. M. (2006). Psicología y salud: algunas reflexiones críticas sobre su qué y su para qué. Universitas Psychologica, 5, 669-679.

Piña, J. A., Ybarra, J. L., Alcalá, I. G., \& Samaniego, R. A. (2010). Psicología y salud (I): la importancia de llamarse modelo y apellidarse teórico-conceptual. Revista Mexicana de Investigación en Psicología, 2, 21-29.

Real Academia Española (1985). Diccionario de la Lengua Española. Barcelona: Océano.

Reidl, L. (2008). Competencias profesionales para los psicólogos. En C. Carpio (Coord.), Competencias profesionales y científicas del psicólo- go: investigación, experiencias y propuestas (pp. 15-42). México: Universidad Nacional Autónoma de México.

Reynoso, L., Álvarez, M. A., Tron, R., de la Torre, I., \& Seligson, I. (2002). Conducta tipo A y enfermedad cardiovascular. En L. Reynoso e I. Seligson (Coords.), Psicología y salud (pp. 43-70). México: Universidad Nacional Autónoma de México/Consejo Nacional de Ciencia y Tecnología.

Ribes, E. (2005). Reflexiones sobre la eficacia profesional del psicólogo. Revista Mexicana de Psicología, 22, 5-14.

Ribes, E. (2006). Competencias conductuales: su pertinencia en la formación y práctica profesional del psicólogo. Revista Mexicana de Psicología, 23, 19-26.

Ribes, E. (2009). Reflexiones sobre la aplicación del conocimiento psicológico: ¿Qué aplicar o cómo aplicar? Revista Mexicana de Análisis de la Conducta, 35, 3-17.

Ribes, E. (2011). La psicología: Cuál, cómo y para qué. Revista Mexicana de Psicología, 28, 8592.

Ribes, E., \& López, F. (1985). Teoría de la conducta: un análisis de campo y paramétrico. México: Trillas.

Rodríguez, G., \& Rojas, M. (1998). La psicología de la salud en América Latina. En G. Rodríguez y M. Rojas (Coords.), La psicología de la salud en América Latina (pp. 14-32). México: Universidad Nacional Autónoma de México/ Miguel Ángel Porrúa.

Sánchez-Sosa, J. J. (1998). Desde la prevención primaria hasta ayudar a bien morir: la interfaz, intervención-investigación en psicología de la salud. En G. Rodríguez y M.E. Rojas (Coords.), La psicología de la salud en América Latina (pp. 33-44). México: Universidad Nacional Autónoma de México/Miguel Ángel Porrúa. 
Sánchez-Sosa, J. J. (2008). Competencias científicas y profesionales: Cimientos metodológicos y de integración en las ciencias del comportamiento. En C. Carpio (Coord.), Competencias profesionales y científicas del psicólogo: investigación, experiencias y propuestas (pp. 247-282). México: Universidad Nacional Autónoma de México.

Sánchez-Sosa, J. J. (2009). El impacto social de la investigación psicológica en salud. Revista de Psicología Social y Personalidad, XXIV, 115-120.
Scopetta, O. (2006). Discusión sobre la evaluación de impacto de programas y proyectos sociales en salud pública. Universitas Psychologica, 5, 695-703.

Stone, G. C. (1988). Psicología de la salud: una definición amplia. Revista Latinoamericana de Psicología, 20, 15-26.

Ybarra, J. L., \& Samaniego, R. A. (2010). Prospectiva de la diabetes tipo 2 en México: aportaciones de la psicología de la salud. En F. López (Comp.), Prospectiva de la psicología de la salud en México (pp. 97-118). México: Consorcio de Universidades Mexicanas. 